\title{
e-Migrinter
}

9| 2012

Immigrés, illégaux, réfugiés. Questions sur les enquêtes et les catégories

\section{Un terrain dit « sensible » dans le champ des migrations : réflexivité sur une expérience marocaine}

\section{Anaik Pian}

\section{(2) OpenEdition}

Journals

Édition électronique

URL : https://journals.openedition.org/e-migrinter/776

DOI : 10.4000/e-migrinter.776

ISSN : 1961-9685

Éditeur

UMR 7301 - Migrinter

Édition imprimée

Date de publication : 20 juillet 2012

Pagination : 79-90

ISSN : 1961-9685

Référence électronique

Anaik Pian, «Un terrain dit « sensible » dans le champ des migrations : réflexivité sur une expérience marocaine », e-Migrinter [En ligne], 9 | 2012, mis en ligne le 11 juillet 2016, consulté le 20 mai 2021.

URL : http://journals.openedition.org/e-migrinter/776 ; DOI : https://doi.org/10.4000/e-migrinter.776 


\section{Un terrain dit « sensible " dans le champ des migrations : réflexivité sur une expérience marocaine}

Anailk Pian

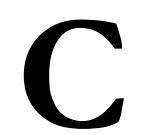

e texte porte un regard réflexif sur une enquête de terrain menée entre 2003 et 2006 au Maroc auprès de Sénégalais cherchant à rejoindre clandestinement l'Europe. Il insiste sur les enjeux et les difficultés de la relation d'enquête : comment travailler en et sur une situation de clandestinité ?
Introduction

Il y a des enquêtes ethnologiques qui marquent plus que d'autres. Se «frotter» à la réalité du terrain, pour reprendre les préceptes de l'École de Chicago ${ }^{1}$, est une expérience à part entière qui demande, à chaque instant, de s'ajuster aux contraintes et opportunités du moment, et de gérer une relation d'enquête qui, à la moindre inadvertance, peut se couvrir de nombreuses ambiguités. Cet article propose de porter un regard réflexif sur une enquête de terrain menée entre 2003 et 2006 au Maroc auprès des aventuriers $^{2}$ sénégalais cherchant à rejoindre clandestinement l'Europe, à défaut de pouvoir s'y rendre légalement. À partir des années 2000, les Sénégalais, comme d'autres migrants subsahariens, sont de plus en plus nombreux à tenter de contourner les

${ }^{1}$ Le «pragmatisme méthodologique » (Réa et Tripier, 2003) de l'École de Chicago donne une place importante à l'observation et à l'observation participante pour saisir les processus sociaux étudiés. Le terrain est premier, la théorie ne préexiste pas à l'enquête. Cette posture est fortement critiquée par Burawoy (2010) en ce qu'elle ne répond pas, selon lui, aux exigences d'une ethnographie globale et historique. Selon Burawoy, l'ethnographie se doit de replacer au premier plan la théorie (qui permet de saisir les «forces globales externes » inscrites dans un processus historique), dans une conception poppérienne de la démarche d'enquête. Si les critiques ainsi adressées à la méthodologie de Chicago ont elles-mêmes suscité des débats (Le Méner, 2010), dans cette étude, la démarche inductive adoptée, donnant le primat au terrain, s'est accompagnée d'une prise en compte du contexte géopolitique dans lequel ces migrations se meuvent, ainsi que de la dynamique historique de la présence sénégalaise au Maroc.

${ }^{2}$ Appellation par laquelle se désignent de nombreux Sénégalais venant au Maroc dans le but de "passer» en Europe. Partant de la subjectivité des acteurs, j’ai par la suite repris ce terme pour rendre compte d'une expérience et d'une carrière migratoire (Pian, 2009). Sur la notion de carrière, cf. Martiniello et Réa (2011). 
politiques d'immigration drastiques des pays européens en cherchant à atteindre l'Espagne - porte d'entrée de l'Europe - à partir du territoire marocain. Si ce texte s'attache à rendre compte de la fabrique de la relation ethnographique, quelques précisions sur le découpage de l'objet apparaissent préalablement nécessaires pour resituer le point de vue méthodologique adopté. En effet, dans une recherche, le choix de l'unité de base de l'objet peut se faire à partir de différents critères, tels le lieu (perspective généralement privilégiée par les géographes) ou le groupe social ${ }^{3}$ (perspective généralement privilégiée par les sociologues), ces deux options pouvant se combiner ou donner lieu à une démarche multi-sites (Marcus, 2010). L'ethnographie multi-située, qui puise son inspiration dans le socle théorique des études post-modernes, instaure une cartographie du terrain pouvant se décliner sous deux formes. L'une d'elle, qualifiée «d'ethnographie uni-site stratégiquement située » implique d'enquêter, outre sur un site principal d'observation, sur des sites connexes à celui-ci. La seconde approche suppose de suivre dans le temps et dans l'espace la circulation des personnes, des biens, des marchandises, etc. qui sont au cœur de l'enquête. Mais ce sont également les routes migratoires en elles-mêmes et les trajectoires de mobilité qui s'y jouent qui peuvent être retenues (Brachet, 2009).

Dans cette étude, la démarche a été celle d'une «ethnographie uni-site» combinée à une focale sur les Sénégalais. En effet, au terme d'un terrain exploratoire mené à Casablanca, le cas des Sénégalais m'est apparu particulièrement intéressant dans la mesure où ces derniers se distinguent par l'ancienneté de leur présence au Maroc, celle-ci étant étroitement liée aux échanges religieux et commerçants, puis estudiantins. De ce fait, ces nouvelles migrations sénégalaises vers l'Europe se greffaient sur tout un système d'échanges et de mobilité

\footnotetext{
${ }^{3}$ Le groupe social lui-même peut également être appréhendé de différentes manières, comme je l'expliciterai un peu plus loin.
}

anciens entre le Sénégal et le Maroc mettant en présence, outre les aventuriers, des étudiants, commerçant(e)s, pèlerins, etc. ${ }^{4}$. Outre Casablanca, l'enquête s'est alors progressivement ouverte à d'autres sites d'investigations, à Rabat, mais aussi, dans une moindre mesure, à Fès. D'une part, parce que ces villes connectent les itinéraires migratoires. D'autre part, parce que la présence des Sénégalais y est relativement importante et diversifiée en termes d'activités et de profils migratoires. Le choix de se centrer sur les Sénégalais - dans différents sites d'enquête - permettait ainsi d'adopter une approche dynamique interrogeant l'évolution d'un champ migratoire sous l'effet d'une conjoncture inédite. Il relevait d'un parti pris méthodologique et analytique de traiter des différentes catégories de mobilité constitutives d'une histoire migratoire, tout en se démarquant d'un point de vue ethnographique uniquement focalisé sur les réseaux de passage à destination de l'Europe. Cette position constructiviste et relationnelle évitait un pré-découpage de l'objet qui, s'alignant de manière trop importante sur les enjeux politico-médiatiques du moment, ne verrait dans le contexte marocain que la seule migration à destination de l'Europe.

Pour reconstruire les ensembles significatifs dans lesquels ces mouvements

${ }^{4}$ Depuis les années 1960, notamment, des commerçants et courtiers sénégalais se sont installés à Casablanca, la capitale économique marocaine. Impliqués dans des réseaux d'import/export, ils assistent les commerçantes sénégalaises qui effectuent des navettes régulières entre Dakar et les places marchandes marocaines, voire des destinations plus lointaines (Dubaï, La Mecque, Italie, etc.). Ces activités prolongent les échanges commerciaux qui, remontant à l'époque précoloniale, accompagnaient les routes religieuses conduisant les fidèles musulmans à Fès et à La Mecque. Au tournant des indépendances, le Maroc accueillit par ailleurs un nombre croissant d'étudiants subsahariens francophones, d'abord dans le cadre de dispositifs étatiques puis, à partir des années 1990, de plus en plus dans le cadre de formations privées. Aujourd'hui, nombre d'entre eux viennent poursuivre leurs études supérieures dans le pays à défaut d'avoir pu obtenir un visa étudiant pour l'Europe ou les États-Unis. 
migratoires se fondent, il apparaissait nécessaire de sortir des cadres préfigurés selon lesquels ces derniers étaient généralement abordés. Comme le rappelle Schwartz (1993, p. 294) à propos de la conception maussienne du « fait social total » (Mauss, 2007), plus généralement transposable à la démarche ethnographique : «La transversalité des objets a des implications positives tant sur leur mode de découpage que sur la manière de les travailler. Elle met en garde contre la constitution d'unités isolées et monofonctionnelles, et recommande une attention spéciale à leur aspect sériel, c'est-àdire à la possibilité qu'ils offrent de circuler entre les multiples sphères et niveaux d'organisation du social ». Aussi, dès lors que les catégories de situation et le passage d'un statut migratoire à un autre sont poreux dans le temps et l'espace de la migration ${ }^{5}$, le suivi longitudinal des parcours impliquait de ne pas pré-définir de manière rigide la population cible de l'étude, à savoir les aventuriers, mais de s'intéresser aux bifurcations et reconversions au cours des carrières migratoires. Ce découpage de l'objet a par ailleurs posé la question de l'exemplarité/spécificité de cette population d'étude par rapport aux autres migrants subsahariens au Maroc. Dans une perspective transversale, les parcours migratoires de ces derniers ont été, d'un point de vue ethnographique, essentiellement abordés à partir des imbrications existantes avec les réseaux migratoires sénégalais.

\section{Les premiers pas du terrain}

Mes recherches empiriques ont débuté à Casablanca où, peu à peu, je me suis familiarisée avec le milieu commerçant sénégalais. Après avoir passé un après-midi à

\footnotetext{
${ }^{5} \mathrm{Un}$ aventurier initialement venu au Maroc pour tenter de rejoindre l'Europe peut se reconvertir plus ou moins durablement en commerçant tandis qu'un étudiant en difficulté financière ou en fin de cursus peut être tenté par le passage clandestin en Europe, etc.
}

arpenter les rues de la capitale économique marocaine sans trop savoir où et comment je pourrais rencontrer des migrants subsahariens, c'est à l'entrée de la vieille Médina que j'ai aperçu des vendeurs de rue sénégalais présentant leurs marchandises sur des étalages de fortune. Après quelques hésitations, je me suis présentée à l'un d'eux en tant qu'étudiante en sociologie. Tout d'abord très méfiant, mon interlocuteur qui, par la suite, deviendra un informateur privilégié, accepte de me revoir le lendemain et me propose de déjeuner dans un restaurant sénégalais. C'est à l'annonce de mon prénom breton que son attitude change subitement. Tout à coup enthousiaste, il m'explique avoir vécu à Rennes, ville qu'il a beaucoup appréciée et où j'ai moi-même vécu. Première surprise du terrain: je n'aurais jamais imaginé que ma première rencontre avec un Sénégalais au Maroc serait facilitée par mon prénom breton!

Une dimension temporelle essentielle a alors encadré ma présence sur le terrain : il s'agit de la quotidienneté. Les sociologues sont nombreux à avoir insisté sur son importance (Simon-Barouh, 1983; Schwartz, 1993). Par la disponibilité qu'elle implique, la quotidienneté entrouvre de nombreuses portes, celles de la reconnaissance et de la confiance qu'elle suscite à terme. Mon terrain a été rythmé par de nombreuses journées passées aux côtés des vendeurs de rue, de nombreux aprèsmidi consacrés à rencontrer des commerçant(e)s ou des étudiants et à les accompagner dans leurs déplacements, de longues heures passées dans un restaurant sénégalais mais aussi de longues heures à attendre, en vain, des rendez-vous ajournés. À chaque instant, j'essayais de faire preuve d'une «attention flottante» (Laplantine, 1996) et d'une "vigilance routinière » (Cefai, 2003), restant attentive aux moindres évènements. Si certains de ces moments se révélaient d'emblée féconds, d'autres donnaient l'impression d'une "perte de temps». Il n'était pas rare, en outre, que les discussions et observations effectuées 
semblaient ne rien apporter à l'analyse ni même à la découverte, car elles restaient d'ordre très général ou ne touchaient pas directement aux thèmes qui motivaient ma recherche. Dans certains cas, je recueillais même des témoignages que je savais inexacts par recoupements avec d'autres données ou d'autres observations. Je ne cherchais pas nécessairement à aborder les questions qui m'intéressaient le plus, préférant une disposition plus favorable de mes interlocuteurs.

C'est ainsi que, d'une manière inattendue, j'ai pu nouer des premiers contacts avec le milieu des aventuriers. Durant mes deux premiers mois de terrain, je n'avais pu obtenir que des propos généraux et évasifs à l'égard de cette migration à destination de l'Europe. Alors que je me demandais combien de temps il faudrait pour lever cette barrière, c'est un appel téléphonique adressé à une Sénégalaise durant mon retour en France qui m'a ouvert, lors de ma seconde venue à Casablanca en février 2004, mes premiers contacts avec le milieu. Comme je le compris par la suite, celle-ci avait elle-même rejoint le Maroc dans l'espoir de parvenir en Europe et vivait dans une de ces maisons où se regroupent les aventuriers. Elle m'y introduisit dans la mesure où mon appel avait été interprété comme une preuve de confiance. C'est par son intermédiaire que j'ai pu effectuer ma première visite à Takadoum, quartier populaire de Rabat où résident, au sein d'habitations collectives communément appelées foyers, de nombreux aventuriers dans l'attente du passage ${ }^{6}$.

Les enjeux de la situation d'enquête eux-mêmes sont apparus révélateurs de règles sociales structurant les réseaux migratoires vers l'Europe. Ainsi, lors de ma première venue dans un foyer à Takadoum, des débats ont divisé les occupants des lieux, en dépit des médiations d'un informateur

${ }^{6} \mathrm{Ces}$ foyers, pour reprendre la dénomination employée par les intéressés, sont en fait des maisons à étage louées à des familles marocaines. privilégié. Des aventuriers étaient opposés à ma présence ou, du moins, la conditionnaient à un droit d'entrée de l'ordre de 50 euros. Cette somme qui, a priori, pouvait être perçue comme une «taxe» appliquée à tout observateur extérieur, souvent renvoyé à l'image du journaliste qui recueille des témoignages contre rémunération avant de "disparaitre", s'inscrivait toutefois dans un contexte de sens plus complexe. En effet, les aventuriers eux-mêmes, doivent s'acquitter d'un «droit d'entrée » pour séjourner dans les foyers. En tant qu'ethnologue, j'étais alors soumise aux mêmes injonctions que les migrants, même si les chefs de foyers concernés ont finalement concédé à une dérogation à la règle. En ce sens, la relation d'enquête ellemême peut contribuer à renseigner sur «l'intelligibilité pratique des échanges» (Althabe, 1990) et des contextes.

\section{Discussions informelles et entretiens :} jouer sur l'entre-deux des positions

D'emblée, un constat s'est imposé : je devais me débarrasser de tout support donnant un côté trop officiel à la recherche. Les méthodes par trop canoniques (entretiens formels accompagnés d'une grille d'entretien), qui constituent des «modes officiels de construction et d'accès à la parole d'autrui» (Laacher, 2004, p. 53), tendaient à alimenter l'auto-censure des discours. C'est pourquoi, de manière parallèle aux observations, j'ai privilégié de nombreuses discussions informelles et répétées que je retranscrivais ensuite sur un journal de bord. Le plus souvent, ce n'est qu'après l'établissement d'une relation de confiance progressivement tissée que je demandais aux intéressés s'ils voulaient bien m'accorder un entretien enregistré. Cette demande n'était pas systématique mais dépendait des circonstances dans lesquelles nous nous côtoyions ainsi que du type de lien établi. En effet, il devenait parfois presque gênant de solliciter un entretien plus formel auprès de personnes faisant office 
d'informateur privilégié ou avec lesquelles une plus grande familiarité s'était établie. Dans quelques cas, j'ai pu solliciter un entretien enregistré dès la première rencontre tout en ayant conscience des biais qui risquaient d'être ainsi accentués. Je considérais alors le matériau recueilli comme une première base et j'apportais un intérêt particulier aux évolutions du discours au cours de rencontres ultérieures. Presque tous les entretiens ont été menés en français, même si la présence d'un tiers jouant le rôle d'interprète a parfois été nécessaire.

Qu'ils soient formels ou informels, les entretiens ont toujours été conduits de façon à instaurer un dialogue le plus naturel possible. Sur les modèles de l'entretien compréhensif (Kaufmann, 1996), il s'agissait d'éviter «un registre de rupture avec la communication ordinaire » (Althabe, 1990) pour, au contraire, mener des entretiens souples tendant à se rapprocher du registre de la discussion. Tenant un carnet dans lequel je notais les scènes, les impressions, les découvertes, mais aussi les questions auxquelles je n'avais pas encore de réponse, je préparais chaque rencontre, ou plutôt chaque journée sur le terrain (car je ne savais pas toujours exactement qui j'allais $y$ rencontrer), en notant les thèmes que je souhaitais aborder l'occasion venue. Je pouvais ainsi me constituer de véritables grilles d'entretien mais celles-ci sont toujours restées d'un usage indirect. Je ne les utilisais jamais de manière visible mais me contentais de les intérioriser, avec la part d'oublis qu'un travail de mémoire en situation d'interaction contient inévitablement. Comme je privilégiais, dans la mesure du possible, des relations sur le long terme, il m'était généralement possible de revenir sur ces omissions par la suite. Lors des retranscriptions, ces entretiens pouvaient apparaitre un peu décousus, mais c'est justement leur caractère non académique qui faisait leur richesse. Ce bricolage permettait d'atténuer la dualité des rôles enquêteur/enquêtés en assouplissant les positions respectives de celui qui questionne et de celui qui répond.

Appelant à évoquer des «récits de pratiques en situation » (Bertaux, 2003), des récits de trajectoires ou encore certains évènements, ces entretiens et discussions ouverts offraient une marge de manœuvre aux intéressés. La démarche adoptée leur laissait la possibilité d'intervenir comme témoin de leur propre expérience ou comme informateur. Mais aussi et surtout, elle leur permettait d'osciller entre l'une et l'autre de ces positions ; et ce qui avait tout d'abord été dit sur le ton d'une information délivrée par un informateur devenait souvent, au cours $\mathrm{du}$ temps, confidence sur son propre itinéraire.

\section{Les effets d'optique du terrain}

Progressivement, j’ai été amenée à élargir mon regard. En dépit de diverses formes d'alliance, il existe une certaine concurrence entre les migrants subsahariens au Maroc. Au terme d'un terrain exploratoire, ce constat a également interféré dans le choix de retenir la population sénégalaise comme population d'étude. En effet, travailler sur la population subsaharienne dans son ensemble posait, outre la pertinence du recours à cette catégorie d'analyse ${ }^{7}$, la question de parvenir à négocier sa position à l'égard de tous, sans se retrouver en porte-à-faux. L'expérience de Rabinow (1988) au Maroc rappelle comment les ethnologues sont fréquemment pris dans des enjeux de catégorisation qui les identifient comme potentiels alliés ou à l'inverse comme «espions» du camp adverse.

Par la suite, je me suis rendue compte que cette concurrence m'était sans doute apparue de manière accentuée du fait que mon terrain s'est tout d'abord déroulé à

${ }^{7}$ En effet, les intéressés se reconnaissent-ils dans cette catégorie? Ou procède t-elle d'une «tentation nominaliste » (Balandier, 1985) imposant une uniformisation catégorielle de l'extérieur? 
Casablanca, où l'enjeu du commerce de rue conduit les Sénégalais à se démarquer, plus jalousement quailleurs, de potentiels concurrents $^{8}$. Les projets migratoires et leurs évolutions influent sur l'activité sociale au sens wébérien et, ce faisant, orientent les relations entretenues ainsi que la modulation des frontières entre les «Eux» et les «Nous». À cela s'ajoutaient les effets d'optique induits par le choix de me centrer sur un groupe prédéfini. Comme le rappelle Timera (1996), la construction de l'objet tend à se décliner autour de deux grandes orientations qui produisent des points de vue différents. Dans un premier cas, le chercheur part d'un groupe circonscrit et analyse ensuite les dynamiques qui le traversent. Afin d'éviter que cette vision centrée occulte les dynamiques extracommunautaires, il ne faut pas oublier que le groupe (qu'il soit construit par les acteurs eux-mêmes ou par le chercheur) n'a de sens que dans le cadre des systèmes d'interaction dans lequel il se meut. Prendre en compte ces considérations permet de dresser un pont envers le second type d'approche méthodologique. À l'inverse de la première posture décrite, celui-ci privilégie un point de vue ancré sur la logique des réseaux. C'est alors l'acteur et non plus le groupe qui se retrouve au centre de l'investigation.

La suite de mon terrain à Rabat, autre relais migratoire clé, m'a permis de mieux recontextualiser ces dimensions relationnelles et méthodologiques, et par làmême, de saisir plus clairement les enjeux y étant liés. Bien que les réseaux migratoires constitués par les différents migrants subsahariens possèdent leurs propres règles de voyage, ces derniers ont développé des créations institutionnelles ad hoc communes en vue de maximiser les chances de réussite du passage (Pian, 2009). La solidarité pragmatique dont relèvent ces formes d'organisations (qui n'excluent pas, cependant, des conflits parfois violents)

\footnotetext{
${ }^{8}$ Aujourd'hui, toutefois, la situation a évolué et ce commerce s'est développé dans d'autres villes marocaines.
}

invite, d'un point de vue analytique, à distinguer le domaine de la relation, de l'ordre des pratiques sociales concrètes, et le domaine de l'identification à un ensemble culturel donné, de l'ordre des représentations. Bien que l'un et l'autre puissent se conforter, ils ne sont pas nécessairement concordants ni exclusifs. L'identification et l'attachement à ses origines (elles-mêmes plurielles et segmentaires) sont une chose; mais elles ne déterminent pas nécessairement l'activité sociale au sens wébérien. On retrouve ici la distinction faite par Levitt et Glick Schiller (2004) entre les ways of belonging et les ways of being. Les premiers procèdent des voies d'appartenance disponibles ou optionnelles que chacun peut s'approprier ou se voir attribuer et qui se puisent dans un panel de positions occupées. Les seconds, en revanche, réfèrent aux voies «d'être » ou de participation concrète dans un champ social donné. Il est fréquent, en effet, de participer et d'entretenir certaines relations dans un champ social sans pour autant s'identifier aux éléments culturels associés à ce dernier.

Ainsi, les aventuriers sénégalais établissaient, à travers leurs discours, une distinction entre les ressortissants subsahariens desquels ils se sentaient «culturellement» proches, à savoir les Maliens, les Guinéens, les Nigériens, les Gambiens mais aussi les Mauritaniens, et les autres, catégorisés sous le terme aak. Cette dénomination qui regroupe avant tout des non-musulmans, désigne les Subsahariens d'Afrique centrale aux mœurs et à l'accent linguistique jugés non seulement distincts, mais d'une distinction dépréciée. Le sens commun leur attribue maints méfaits (pratiques alimentaires peu orthodoxes, propagation du sida, etc.). Inversement, les Congolais tenaient des propos souvent très critiques à l'égard des Ouest-Africains en mettant en cause, à travers une rhétorique essentialisante, leur mentalité et comportements jugés "naturellement » grossiers ou peu fiables. Si des différences de position sociale au pays d'origine pouvaient 
influer sur ces logiques dépréciatives ${ }^{9}$, cellesci pouvaient également être renforcées par les effets de la situation d'enquête. Comme le rappelle Schwartz (1993), la dévalorisation de l'autre peut constituer un moyen de « faire alliance » avec le chercheur et, par làmême, se valoriser auprès de lui. Ainsi, il n'était pas rare que des Sénégalais soulignent, à travers des arguments essentialistes les différenciant des aakes, leur attitude particulièrement respectueuse à l'égard des femmes. Inversement, les Congolais ne manquaient pas de fustiger le comportement des Africains de l'Ouest à l'égard de la gente féminine. Dénonçant leur machisme et la pratique de la polygamie, ils en attribuaient une part de responsabilité à leur filiation musulmane alors renvoyée à une véritable «nature » se transmettant par les gènes. Ces propos prenaient sans doute une teneur particulière en ma présence où chacun essayait de valoriser son attitude envers les femmes, mais aussi de me décliner sa conception occidentalisée du couple.

\section{Le poids des identifications}

Afin de ne pas me laisser submerger par le terrain, un effort constant de contextualisation de la relation aux personnes rencontrées a été nécessaire. Deux interrogations revenaient de manière récurrente: à qui les aventuriers croyaient-ils s'adresser et qu'attendaient-ils des liens qui s'instauraient entre nous ? Tout d'abord, ces derniers avaient légitimement besoin de me situer dans ma relation à eux. Le rapport dominant/dominé ne pouvait être ignoré. Il ne leur était pas si facile, en effet, de confier vouloir « passer » en Europe à quelqu'un qui, justement, appartenait à cette société située de «l'autre côté ». Ainsi, la connaissance de ma position à l'égard de la politique migratoire européenne conditionnait leur parole et l'acceptation de me recevoir. Si Favret-Saada (1999) souligne qu'en ethnologie des sorts, il n'y a pas de place

\footnotetext{
${ }^{9} \mathrm{Au}$ Maroc, les Congolais qui cherchent à atteindre l'Europe ont globalement un niveau d'étude supérieur aux autres migrants subsahariens.
}

pour un ethnologue non impliqué, un même constat prévaut en sociologie des migrations. $\mathrm{Au}$ cours du terrain, les deux principales difficultés auxquelles je me suis heurtée ont tenu à l'identité et au statut qui m'étaient assignés, l'un en tant que femme européenne, l'autre tenant à une confusion souvent faite a priori et qui tendait à m'assimiler à une journaliste. En tant que toubab ${ }^{10}$, tout d'abord, il n'a pas toujours été facile de négocier ma présence ou, plutôt, de lever les ambiguiités qui n'avaient de cesse de surgir. Certains aventuriers voyaient en moi la possibilité d'un financement de la traversée clandestine ou encore d'un mariage qui pourrait les faire accéder à l'Europe. Le fait qu'au début de mon terrain, j'ai été introduite dans le milieu par une Sénégalaise qui se prostituait pour subvenir à ses besoins (Pian, 2010), a sans doute influencé le regard que ces derniers pouvaient porter sur moi. En outre, dans un monde où presque tout se vend et se marchande, négocier ma présence n’a pas toujours été évident.

Agier (2005) soulève les enjeux méthodologiques tenant à la présence, de plus en plus fréquente sur les terrains d'enquête, d'un "troisième élément » s'interposant (directement ou non) entre le chercheur et la population d'étude. Comme le constate Goldschmidt (2005) lors de ses investigations auprès des Congolais à Rabat, il s'agit souvent d'acteurs associatifs intervenant sur le même terrain que le sociologue. Cependant, pour ma part, cette dimension a peu interféré dans les investigations menées. En effet, jusqu'en 2005, les Sénégalais étaient très peu nombreux à solliciter des aides auprès des quelques structures caritatives et religieuses (Caritas, les Eglises évangéliques) apportant un certain soutien moral et matériel à cette population subsaharienne. Aujourd'hui, la donne serait certainement différente puisque

\footnotetext{
${ }^{10}$ Terme utilisé par les Sénégalais pour désigner les Occidentaux. Pour plus de précisions sur les usages et l'historique de cette figure, cf. Fouquet (2009) et Doquet (2005).
} 
depuis les évènements de Ceuta et Melilla ${ }^{11}$, les associations intervenant auprès de ces migrants se sont multipliées, qu'elles soient engagées dans des actions juridiques de défense de leurs droits ou d'aide humanitaire.

Pour ma part, cette «triangulation de l'enquête » (Agier, 2005) tenait beaucoup plus à la figure du journaliste. En raison, sans doute, des effets pervers de la surmédiatisation de certains sujets et du type de relation qu'elle impliquait (relation ponctuelle et de courte durée, le plus souvent sans retour), cette identification alimentait des discours plats et stéréotypés. Les ressemblances dans les méthodes de recueil de données en journalisme et en sociologie concouraient à entretenir cette confusion d'autant plus que, généralement, les enquêtés se représentaient assez mal ce que pouvait être la sociologie. En outre, la restriction du formalisme d'enquête qui s'imposait pour déconstruire cette assimilation à une journaliste laissait, en retour, la porte ouverte aux diverses ambiguités quant à ce que les aventuriers attendaient - ou pouvaient attendre - de moi. Pour sortir de ce dilemme, c'est un équilibre en tension entre ces différentes identifications qu'il m'a fallu réussir à déconstruire, puis à maintenir et à gérer : «(...) chaque rôle social est un ensemble complexe de distances " note à cet égard Hughes (1996). Entre la familiarité qui s'instaurait et la part d'étrangeté qui persistait, je devenais parfois une confidente privilégiée auprès des aventuriers. "L'altérité reconnue » ou cette part de distance irréductible liée à ma position (étudiante, chercheuse, femme française) a permis d'instaurer une certaine relation de proximité donnant accès à des «paroles privées" (Schwartz, 1993). Ma présence assouvissait parfois un besoin de se confier et d'exprimer découragements et espoirs, dans un milieu

11 À l'automne 2005, quelques milliers de migrants tentaient de franchir les grillages de Ceuta et Melilla, protégeant les enclaves espagnoles situées sur le territoire marocain. très masculin où les aventuriers pouvaient difficilement faire part de leurs doutes à leurs compagnons de route, tandis que leur famille au pays ignorait le plus souvent leurs conditions de vie ${ }^{12}$. L'extériorité de ma position devenait ainsi condition de proximité, ce qui a alors soulevé la question du «don contre don», d'autant plus prégnante lorsque le chercheur se retrouve face à des situations de grande précarité (sur ce point, $c f$. Pian 2009 et 2012).

\section{Territoires de l'enquête : clandestinité et visibilité contrainte du chercheur}

Le chercheur qui mène une étude auprès de personnes vivant en situation irrégulière porte une grande responsabilité : avant toute chose, il doit veiller à ce que ses déplacements ne soient pas préjudiciables aux intéressés. J'ai pleinement pris conscience de ces enjeux lors d'une journée passée à Takadoum, quartier populaire de Rabat où vivent de nombreux aventuriers au sein de foyers. L'un d'eux, un Sénégalais, était venu me chercher à l'entrée du quartier puis m'y avait reconduite en début de soirée. Or, à peine étais-je montée dans un taxi pour regagner la gare, qu'il s'est fait contrôler par un policier en civil. Étant en situation irrégulière, il aurait pu se faire expulser à la frontière algérienne si le policier n'avait pas fait preuve du laxisme finalement concédé. Protestant vivement, mon accompagnateur arguait qu'il était un étudiant de Casablanca et qu'il avait oublié de se munir de ses papiers.

À Takadoum, en 2004-2005, la venue d'une jeune femme blanche ne pouvait qu'attirer l'attention en suscitant des regards pesants, oppressants et inquisiteurs. Malgré les dispositions prises pour essayer de passer inaperçue, je ne pouvais pas l'être. À cela,

\footnotetext{
${ }^{12}$ Dans son enquête ethnologique sur les vendeurs de journaux dans le métro, Stettinger (2003) témoigne également de la «demande affective » émanant de la part des enquêtés, et qu'elle a dû parvenir à gérer.
} 
s'ajoutait parfois la désapprobation des propriétaires ou concierges marocains de me laisser pénétrer dans des maisons où vivaient des aventuriers. À plusieurs reprises, mes accompagnateurs ont dû me présenter comme une amie de leurs parents afin de vaincre leur réticence. Si cela pouvait suffire à dissiper leur méfiance, en d'autres circonstances, la défiance restait de mise. De même, à Casablanca, l'hôtesse d'accueil d'un petit hôtel pension, réputé pour abriter des aventuriers, refusait catégoriquement de me laisser rentrer dans l'établissement. Afin de moins attirer l'attention, de nombreux rendez-vous se sont alors déroulés dans des cafés. Ces lieux anonymes présentaient plusieurs avantages. Paradoxalement peutêtre, ces rencontres attiraient moins le regard $^{13}$ que mes déplacements dans les habitations; de plus, en raison des conditions de colocations entre aventuriers, les discussions pouvaient s'y dérouler de manière plus personnelle. À plusieurs reprises, en outre, des entretiens menés dans des foyers ont été interrompus par des mouvements de panique suscités par des rumeurs de rafles prévues pour le lendemain. Ces conditions d'enquête particulières m'ont permis de mieux saisir les tensions perpétuelles dans lesquelles vivent les aventuriers au Maroc. À mon tour, je me sentais épiée dans mes moindres faits et gestes, même si la différence de nos statuts appelait à ne pas sombrer dans une «illusion fusionnelle » (Olivier de Sardan, 2000).

\section{Conclusion}

Bouillon, Fresia et Tallio (2005, p. 1415) définissent les terrains sensibles à l'aune de trois dimensions principales, qui relèvent tant de la relation aux enquêtés, de la méthodologie de terrain que des enjeux socio-politiques soulevés par l'enquête et la restitution des données : «Nous donnons un

\footnotetext{
${ }^{13} \mathrm{En}$ certaines circonstances, toutefois, cela n'empêchait pas que le vendeur de cigarettes au détail vienne s'asseoir, comme par hasard, à la table voisine de celle où mon interlocuteur et moi, nous étions installés pour discuter!
}

triple sens au qualificatif sensible. En premier lieu, nous le disions, les terrains sont sensibles en ce qu'ils sont porteurs d'une souffrance sociale, d'injustice, de domination et de violence. En second lieu ils sont sensibles parce qu'ils impliquent de renoncer à un protocole d'enquête par trop canonique (...) pour inventer, avec un souci permanent de rigueur, de nouvelles manières de faire. Les espaces enquêtés sont souvent isolés, fermés, à la marge et le caractère éphémère de certaines situations est susceptible de précipiter la recherche, ou de la clore. (...). En dernier lieu, ces terrains s'avèrent sensibles en ce qu'ils relèvent d'enjeux sociopolitiques cruciaux, en particulier vis-à-vis des institutions sociales normatives $»$.

Si l'on s'en tient à cette définition, le terrain marocain présente bel et bien des aspects sensibles. Sensible, tout d'abord, du point de vue de la fabrique de la relation ethnographique, en tant que relation sociale (Papinot, 2010) où les processus d'identification en jeu, instituant des rapports de hiérarchisation et de classifications sociales, interfèrent dans les dimensions sociales et spatiales du terrain. Bien que ce point n'ait pu faire l'objet d'un développement à part entière, cette fabrique de la relation ethnographique pose également les problématiques de «l'activisme circonstancié » (Marcus, 2010) et de l'engagement du chercheur (Cefai, 2010a et b ; Hopper, 2010), celles-ci étant bien plus complexes que le seul « don contre don » se jouant dans le face-à-face avec les enquêtés.

Terrain sensible, ensuite, dans le rapport à un contexte institutionnel (et médiatique) soulevant la question du «droit à enquêter » (Laurens et Neyrat, 2010) et des effets de l'enquête, qu'il s'agisse de l'accès au terrain ou de la restitution des données.

Terrain sensible, aussi, du point de vue du caractère labile et incertain des situations migratoires étudiées, et avec lequel doit composer la temporalité ethnographique. À 
cet égard, si la présence prolongée et quotidienne du chercheur sur le terrain est régulièrement rappelée comme étant constitutive de la démarche ethnographique, j'insisterai pour ma part sur la double temporalité de ce type d'enquête. À côté d'une temporalité longue, s'inscrivant dans la durée (celle-ci étant également marquée par le temps de l'attente, des rendez-vous ajournés, etc.), s'expérimente une temporalité beaucoup plus prompte, faite de réajustements permanents, d'imprévus, de découvertes soudaines, de remises en cause multiples. Rentrer dans la temporalité du terrain, c'est alors s'imprégner du rythme des préoccupations des enquêtés et plus largement des contraintes temporelles du monde social étudié qui, parfois, peuvent entrer en tension avec les contraintes temporelles et institutionnelles de la recherche.

Dimension sensible du terrain, enfin, du point de vue d'une échelle d'observation (Vulbeau, 2007) attentive aux détails du quotidien et des pratiques des acteurs.

Ainsi définis, de nombreux terrains, et notamment ceux qui s'inscrivent dans une ethnographie de la mondialisation, dont relèvent beaucoup d'études sur les migrations (Costey et Gardella, 2010), présentent un caractère sensible. Ces conditions sensibles, cependant, ne semblent pas en soi, fondamentalement nouvelles. Ce qui est sans doute plus marquant, c'est l'intérêt actuel qui leur est porté, à travers une réflexivité méthodologique informant la fabrique du terrain.

Anaik Pian

Sociologue / Post-doctorante Université de Bretagne Occidentale Agence Régionale de Santé anaikpian@yahoo.fr

\section{Bibliographie}

Althabe, Gérard (1990) Ethnologie du contemporain et enquête de terrain, Terrain, $\mathrm{n}^{\circ} 14$, [en ligne, réf. du 15/01/2012] URL: $<$ http://terrain.revues.org/document2976.h $\underline{\operatorname{tm}>}$

Agier, Michel (2005) Ce qui rend les terrains sensibles... et l'anthropologie inquiète, in F. Bouillon; M. Fresia et V. Tallio (eds), Terrains sensibles: expériences actuelles de l'anthropologie, Paris, Ehess, pp. 175-184.

Balandier, Georges (1985) Anthropo-logiques, Paris, PUF, 258 p.(Biblio/Essais).

Bouillon, Florence; Fresia, Marion; Tallio Virginie (2005) Introduction. Les terrains sensibles à l'aune de la réflexivité, in $\mathrm{F}$. Bouillon; M. Fresia et V. Tallio (eds.), Terrains sensibles: expériences actuelles de l'anthropologie, Paris, Ehess, pp. 13-28.

Bertaux, Daniel (2003) Les récits de vie, Paris, Nathan, 127 p. (Collection 128).

Brachet, Julien (2009) Migrations transsahariennes: vers un désert cosmopolite et morcelé au Niger, Paris, Le Croquant, 322 p. (Terra).

Burawoy, Michael (2010) Revisiter les terrains: esquisse d'une théorie de l'ethnographie réflexive, in D. Cefai (ed.), L'engagement ethnographique, Paris, Ehess, pp. 295-351.

Cefai, Daniel (2010a) Présentation du chapitre 8. Un pragmatisme ethnographique: l'enquête coopérative et impliquée, in D. Cefai (ed.), L'engagement ethnographique, Paris, Ehess, pp. 447-472.

Cefai, Daniel (2010b) Présentation du chapitre 9. Codifier l'engagement ethnographique?, in D. Cefai (ed.), L'engagement ethnographique, Paris, Ehess, pp. 493-512.

Cefai, Daniel (2003) Postface. L'enquête de terrain en sciences sociales, in D. Cefai (ed.), L'enquête de terrain, Paris, La Découverte, pp. 467-604. 
Costey, Paul ; Gardella, Edouard (2010) Présentation du chapitre 6. De la poétique de l'ethnographie à une anthropologie globale, in D. Cefai (ed.), L'engagement ethnographique, Paris, Ehess, pp. 353-370.

Doquet, Anne (2005) Tous les toubabs ne se ressemblent pas : les particularités nationales des étrangers vues par les guides touristiques maliens, in GEMDEV et Université du Mali, Mali - France: Regards sur une bistoire partagée, Paris, Karthala, pp. 243-258.

Favret-Saada, Jeanne (1999) Les mots, la mort, les sorts, Paris, Gallimard, $332 \mathrm{p}$.

Goldschmidt, Elie (2005) Enquête institutionnelle et "contre-enquête" anthropologique: migrants en transit au Maroc, in F. Bouillon; M. Fresia et V. Tallio (eds), Terrains sensibles: expériences actuelles de l'anthropologie, Paris, Ehess, pp. 145-174.

Fouquet, Thomas (2009) Migrations et "glocalisation» dakaroises, in M.-C Diop (ed.), Le Sénégal des migrations, Paris, Karthala, pp. 241-276.

Hopper, Kim (2010) De l'ethnographie à l'engagement: les limites du témoignage pour les sans-abris, in D. Cefai (ed.), L'engagement ethnographique, Paris, Ehess, pp. 473-492.

Hughes, E.C. (1996) Qui étudie qui ?, in J.-M. Chapoulie, Le regard sociologique: essais choisis. Paris, Ehess, pp. 301-317.

Kaufmann, Jean-Claude (1996) L'entretien comprébensif, Paris, Nathan, 127 p. (Collection 128).

Laacher, Smain (2004) L'indice comme abrégé du monde, Terrain et Travaux, $\mathrm{n}^{\circ} 7$, pp. 44-54.

Laplantine, François (1996) La description ethnographique, Paris, Nathan, 128 p. (Collection $128 ; n^{\circ} 119$ ).

Laurens, Sylvain; Neyrat, Frédéric (eds) (2010) Enquêter de quel droit? Menaces sur l'enquête en sciences sociales, Paris, Le Croquant, $320 \mathrm{p}$.
Le Méner, Erwan (2010) Présentation du chapitre 5. Globaliser, revisiter: ethnographie, macro-sociologie et histoire, in D. Cefai (ed.), L'engagement ethnographique, Paris, Ehess, pp. 273-294.

Levitt, Peggy; Glick Schiller, Nina (2004) Transnational perspectives on migration: conceptualizing simultaneity, International Migration Review, vol. 38, n¹45, pp. 595-629.

Marcus, E. Gorge (2010) Ethnographie $\mathrm{du} /$ dans le système monde: l'émergence d'une ethnographie multisituée, in D. Cefai (ed.), L'engagement ethnographique, Paris, Ehess, pp. 371-395.

Martiniello, Marco ; Rea, Andréa (2011) Des flux migratoires aux carrières migratoires, SociologieS, [en ligne, réf. du 02/04/2012] URL :

$<\underline{\text { http://sociologies.revues.org/3694 }>~}$

Mauss, Marcel (2007) Essai sur le don. Forme et raison de l'échange dans les sociétés archä̈ques, Paris, PUF, 248 p.

Olivier de Sardan, Jean-Pierre (2000) Le 'Je' méthodologique : implication et explication dans l'enquête de terrain, Revue française de sociologie, $\mathrm{n}^{\circ}$ 41-3, pp. 417-445.

Papinot, Christian (2010) Epistémologie de la démarche de recherche en sciences sociales: quel dépassement possible du modèle positiviste, in J. Hamel (ed.), L'analyse qualitative interdisciplinaire, Paris, L'Harmattan, pp. 203-234.

Pian, Anaik (2012) Le corps-mendiant des migrant-e-s subsaharien-ne-s au Maroc : contourner la racisation par l'affichage de la foi musulmane, Corps, n¹0, pp. 163-172.

Pian, Anaik (2010) La migration empêchée et la survie économique: services et échanges sexuels des Sénégalaises au Maroc, Cahiers du genre, $\mathrm{n}^{\circ} 49$, pp. 183-202.

Pian, Anaik (2009) Aux nouvelles frontières de l'Europe: l'aventure incertaine des Sénégalais au Maroc, Paris, La Dispute, 237 p.

Rabinow, Paul (1988) Un ethnologue an Maroc, Paris, Hachette, 143 p.

Réa, Andréa; Tripier, Maryse (2003) Sociologie de l'immigration, Paris, La Découverte, $122 \mathrm{p}$. 
Schwartz, Olivier (1993) L'empirisme irréductible, postface à l'édition française, in N. Anderson, Le Hobo : sociologie du sans-abri, Paris, Nathan, pp. 265-308.

Simon-Barouh, Ida (1983) Relations interethniques et problèmes de minorités : quelques remarques méthodologiques, Sociologie et Sociétés, vol. XV, n², pp. 155-165. Stettinger, Vanessa (2003) Funambules de la précarité, Paris, PUF, 203 p.
Timera, Mahamet (1996) Les Soninké en France: d'une bistoire à l'autre, Paris, Karthala, $244 \mathrm{p}$.

Vulbeau, Alain (2007) L'approche sensible des quartiers « sensibles", Informations sociales, vol. $5, \mathrm{n}^{\circ} 141$, pp. 8-13. 